\section{Influência da posição de parto vaginal nas variáveis obstétricas e neonatais de mulheres primíparas}

\section{The influence of vaginal birth position on obstetric and neonatal variables in primiparous women}

\section{Abstract}

Objectives: to determine the prevalence of obstetric factors associated with vaginal birth position (VBP)- vertical or horizontal; to investigate correlations between VBP and obstetric factors and neonatal characteristics.

Methods: a cross-sectional study was carried out. The sample comprised 176 primiparous women who had given birth vaginally, between july 2006 and february 2007. The correlation was investigated between VBP and the following obstetric variables: occurrence and severity of spontaneous perineal lacerations, episiotomy, perineal suture, the use of oxytocin and surgical instruments. The newborns were characterized according to gestational age, weight, height, head circumference and one- and fiveminute Apgar. The chi-squared test was used to investigate the correlation between VBP and obstetric variables and Student's t-test to investigate differences in neonatal characteristics according to VBP.

Results: no association was found between VBP and perineal suture, perineal laceration, the use of oxytocin, episiotomy or the use of surgical instruments (forceps or vacuum-extractor). There was a correlation between VBP and episiotomy and a greater prevalence of episiotomy for the horizontal position. VBP had no influence on the characteristics of the newborn.

Conclusions: episiotomy was more prevalent in cases of horizontal birth position, although both birth positions are satisfactory as far as the newborn is concerned.

Key words Natural childbirth, Humanizing delivery
Sabrina Mattos Baracho 1

Elyonara Mello de Figueiredo 2

Lucas Barbosa da Silva 3

Izadora Carolina Araújo Gonçalves Cangussu 4

Daniele Nunes Pinto 5

Elza Lúcia Baracho Lotti de Souza 6

Agnaldo Lopes da Silva Filho 7

1,6,7 Grupo de Pesquisa em Disfunções do Assoalho Pélvico, Hospital das Clínicas. Universidade Federal de Minas Gerais. Belo Horizonte, MG, Brasil.

2,4,5 Escola de Educação Física, Fisioterapia e Terapia Ocupacional. Departamento de Fisioterapia. Universidade Federal de Minas Gerais. Av. Antônio Carlos, 6627, Campus Pampulha. Belo Horizonte, MG, Brasil. CEP: 31270-901. E-mail: elyonara@ufmg.br

3 Hospital Sofia Feldman. Belo Horizonte, MG, Brasil.

\section{Resumo}

Objetivos: determinar a prevalência de fatores obstétricos associados à posição de parto vaginal $(P P V)$ - vertical ou horizontal; investigar correlações entre PPV e fatores obstétricos, bem como sua influência sobre as características neonatais.

Métodos: foi realizado um estudo de corte transversal. A amostra foi composta por 176 mulheres primíparas que realizaram parto vaginal, entre julho/2006 e fevereiro/2007. Foi investigada a correlação entre PPV e as seguintes variáveis obstétricas: ocorrência e grau de laceração perineal espontânea, episiotomia, sutura perineal, uso de ocitocina e instrumentação cirúrgica. Os neonatos foram classificados quanto à idade gestacional, peso, estatura, perímetro cefálico e Apgar $1^{\circ}$ e $5^{\circ}$ minutos. Teste quiquadrado foi aplicado para investigar correlação entre PPV e variáveis obstétricas e o teste t-student para investigar a influência da PPV nas características neonatais.

Resultados: não foi observada correlação entre PPV e sutura perineal, laceração perineal, uso de ocitocina, episiotomia e utilização de instrumentação cirúrgica (uso de fórceps ou vácuo-extrator). Houve correlação entre PPV e episiotomia e maior prevalência de episiotomia na posição horizontal. Não houve influência da PPV nas características neonatais.

Conclusões: houve maior ocorrência de episiotomia na posição de parto horizontal, embora ambas as posições de parto tenham sido satisfatórias para os neonatos.

Palavras-chave Parto normal, Parto humanizado 


\section{Introdução}

Aproximadamente $85 \%$ das mulheres que realizam parto vaginal sofrem algum tipo de trauma perineal, incluindo lesões das estruturas de suporte de tecido conectivo da pelve, das estruturas vasculares, dos nervos e/ou músculos ou diretamente do trato urinário. ${ }^{1}$ Esses traumas são resultantes de lacerações perineais espontâneas ou de episiotomia. 2,3 Tais lesões podem levar, em curto prazo, ao aumento da perda sanguínea, à necessidade de sutura e à dor perineal no pós-parto. ${ }^{4}$ Além disso, o trauma perineal decorrente do parto vaginal tem sido apontado como importante fator de risco para o surgimento de disfunções do assoalho pélvico, incluindose as incontinências urinária e fecal e os prolapsos dos órgãos pélvicos.4,5 Tais disfunções são responsáveis por altos custos para o sistema de saúde e por impacto negativo na qualidade de vida dessas mulheres. 6

O risco de ocorrência de trauma perineal durante o parto vaginal é multifatorial. Fatores obstétricos e neonatais, tais como a realização de episiotomia, segundo estágio do trabalho de parto prolongado, uso de ocitocina, parto vaginal assistido a fórceps ou vácuo-extrator, recém-nascido com peso superior a $4000 \mathrm{~g}$ e nuliparidade estão associados à ocorrência de lacerações perineais. 5,7 Alguns desses fatores não são passíveis de intervenção como, por exemplo, o peso do recém-nascido ou a nuliparidade. No entanto, outros são modificáveis e condutas alternativas têm sido propostas para minimizar os possíveis efeitos negativos do parto vaginal sobre as estruturas do assoalho pélvico. 8

A posição vertical da mulher em relação à horizontal durante o parto vaginal parece constituir um desses fatores por abreviar o período expulsivo. 8,9 Benefícios dessa posição incluem o efeito facilitador da força da gravidade, maior eficiência e intensidade na força de contração uterina, a redução do risco de compressão aorto-cava e consequente melhor oxigenação fetal na fase expulsiva do trabalho de parto, e aumento dos diâmetros pélvicos ântero-posterior e transverso nas posições de cócoras e ajoelhada. ${ }^{10,11}$ Além disso, a verticalização parece oferecer maior conforto às parturientes e um melhor intercâmbio materno-fetal, diminuindo o risco de asfixia fetal. ${ }^{12}$

Atualmente, o Ministério da Saúde do Brasil preconiza a assistência humanizada ao parto, de modo que a mulher deve conduzir seu próprio processo de parto, respaldada por orientações fundamentadas em informações válidas e confiáveis acerca dos possíveis benefícios, relativos ao bemestar materno e fetal, das diferentes posições maternas adotadas durante a fase expulsiva do trabalho de parto vaginal. 13 No entanto, ainda são escassos os dados sobre as características das mulheres brasileiras que realizaram partos vaginais verticais com sucesso, e sobre a relação desses com ocorrências obstétricas relevantes para a função do assoalho pélvico das parturientes assim como para a saúde de seus neonatos.

O presente estudo objetivou identificar a associação entre a posição de parto vaginal e a presença e grau de laceração perineal espontânea, de episiotomia, de sutura perineal, uso de ocitocina e de instrumentação cirúrgica em mulheres primíparas. Além disso, buscou investigar a associação entre a posição de parto vaginal horizontal ou vertical e as características dos neonatos.

\section{Métodos}

Trata-se de um estudo observacional, retrospectivo, de corte transversal, em que foi feito levantamento e análise dos dados de mulheres primíparas que realizaram parto vaginal, no período de julho de 2006 a fevereiro de 2007, no Centro de Parto Normal (CPN) Doutor David Capistrano Filho, do Hospital Sofia Feldman, uma instituição filantrópica ligada ao Sistema Único de Saúde que presta assistência humanizada ao parto, localizada em Belo Horizonte, Minas Gerais, Brasil.

Foram incluídos dados de mulheres primíparas com idades entre 18 e 40 anos, com feto único, idade gestacional entre 37 e 40 semanas, que realizaram parto vaginal. Foram excluídos dados de mulheres com desordens neurológicas e história de aborto prévio. As participantes elegíveis foram identificadas a partir do livro de registros do setor de enfermagem do CPN. As variáveis dependentes do estudo foram: presença e grau de laceração perineal espontânea, realização de episiotomia e de sutura perineal, uso de ocitocina e de instrumentação cirúrgica (fórceps ou vácuo-extrator); idade gestacional, peso, estatura e perímetro cefálico do neonato e Apgar no $1^{\circ}$ e $5^{\circ}$ minutos. A variável laceração perineal espontânea foi categorizada de acordo com os critérios estabelecidos pelo Royal College of Obstetricians and Gynaecologists: 14 laceração de $1^{\circ}$ grau (lesão de mucosa), de $2^{\circ}$ grau (lesão envolvendo a musculatura perineal, mas não o esfíncter anal), de $3^{\circ}$ grau (lesão envolvendo o complexo esfincteriano anal) e de $4^{\circ}$ grau (lesão envolvendo o complexo esfincteriano anal e a mucosa retal). A posição de parto vaginal constituiu a variável independente do estudo e foi categorizada, de acordo com Soong e Barnes 8 como 
vertical (sentada, semissentada, cócoras e de joelhos/Gaskin) ou horizontal (decúbito dorsal ou litotomia, decúbito lateral esquerdo).

Análise descritiva, através de medidas de tendência central e dispersão, foram aplicadas para variáveis intervalares, e através de proporção e frequência para variáveis nominais. Estatística inferencial, através de análise bivariada (teste quiquadrado), foi aplicada para investigar correlação entre posição de parto vaginal (variável independente) e ocorrências obstétricas (variáveis dependentes). $\mathrm{O}$ teste $\mathrm{t}$-student foi usado para investigar a influência da posição de parto (variável independente) sobre as características dos neonatos (variáveis dependentes). Para todas as análises inferenciais foi considerado o nível de significância $(\alpha)$ igual a 0,05 . O cálculo amostral foi feito de acordo com a análise de poder estatístico para o teste quiquadrado.15 Para tal, assumiu-se uma força média de correlação entre a variável independente posição de parto e cada variável dependente investigada; desta forma foram considerados dois graus de liberdade (associação bivariada), nível de significância igual a $5 \%$, força de correlação de 0,3 e poder estatístico de 0,95. O tamanho da amostra foi então definido em 172 participantes. Todas as análises estatísticas foram realizadas através do pacote estatístico StatView.

Essa pesquisa foi aprovada pelo Comitê de Ética em Pesquisa (COEP) do Hospital Sofia Feldman, sob o parecer número 10/2006.

\section{Resultados}

Durante o período de julho de 2006 a fevereiro de 2007, foram registrados dados de 191 mulheres primíparas que realizaram parto vaginal. Desses, 15 prontuários não especificaram a posição de parto vaginal, sendo excluídos da análise. Foram investigados, portanto, os dados de 176 mulheres primíparas que realizaram parto vaginal nas posições horizontal ou vertical. A idade média das mulheres foi de 22,8 anos ( $D P=4,4$ anos), a mediana foi de 22 anos e a moda de 19 anos. Dos 176 partos vaginais ocorridos, $94(55,4 \%)$ foram horizontais e $82(44,6 \%)$ verticais.

Com relação à prevalência das variáveis obstétricas, a laceração perineal espontânea, documentada para 166 mulheres, ocorreu em 103 casos (62\%), sendo 62 casos $(37,4 \%)$ de lacerações de $1^{\circ}$ grau, 39 casos $(23,4 \%)$ de $2^{\circ}$ grau e dois casos $(1,2 \%)$ de lacerações de $3^{\circ}$ grau (Figura 1). Não foram registradas ocorrências de laceração perineal de $4^{\circ}$ grau. Com relação à distribuição da ocorrência de laceração perineal, foram documentados 47 casos $(50 \%)$ em mulheres que realizaram parto horizontal e 56 casos (68\%) em mulheres que realizaram parto vertical. (Tabela 1)

A episiotomia, documentada para 168 mulheres, ocorreu em 19 casos (11\%), sendo 17 (18\%) em

\section{Tabela 1}

Distribuição das ocorrências obstétricas relativas à posição de parto vaginal e suas associações com as posições de parto horizontal e vertical, em 176 mulheres.

\begin{tabular}{|c|c|c|c|c|c|}
\hline Variáveis obstétricas & $\mathrm{n}$ & $\%$ & $\mathrm{n}$ & $\%$ & $p$ \\
\hline Realização de episiotomia & 17 & 18,0 & 2 & 2,5 & $<0,001$ \\
\hline Uso de ocitocina & 39 & 41,4 & 30 & 36,6 & 0,4 \\
\hline Presença de laceração perineal & 47 & 50,0 & 56 & 68,0 & 0,3 \\
\hline Presença de sutura perineal & 49 & 52,1 & 49 & 59,8 & 0,6 \\
\hline
\end{tabular}


Figura 1

Ocorrências ou não de lacerações perineais espontâneas de acordo com as posições de parto documentadas para 166 mulheres. Posição horizontal (decúbito dorsal, lateral e litotomia); posição vertical (cócoras, gaskin, sentada e semissentada).

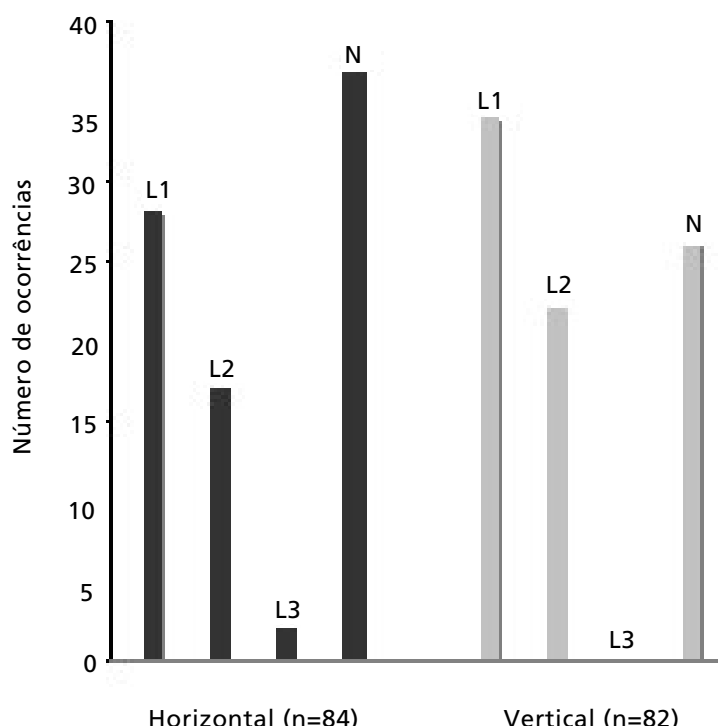

L1=laceração de $1^{\circ} \mathrm{grau} ; \mathrm{L} 2=$ laceração de $2^{\circ} \mathrm{grau}$; L3=laceração de $3^{\circ}$ grau; $N=$ não ocorrência de laceração. partos horizontais e $2(2,5 \%)$ em partos verticais, todas mediolaterais. A episiotomia ocorreu com maior frequência na posição de decúbito dorsal, sendo realizadas $15(88,2 \%)$ nessa posição (Tabela $1)$.

A sutura perineal, documentada para 156 mulheres, ocorreu em 98 (63\%) casos, sendo 49 $(52,1 \%)$ em mulheres que realizaram partos horizontais e $49(59,8 \%)$ em partos verticais (Tabela 1$)$.

Informações sobre uso de ocitocina foram documentadas para 174 mulheres. Sessenta e nove mulheres $(40 \%)$ receberam ocitocina, sendo 39 $(41,4 \%)$ em partos horizontais e $30(36,6 \%) \mathrm{em}$ partos verticais (Tabela 1). Com relação à instrumentação cirúrgica, foi registrado somente um caso de uso de fórceps, ocorrido em parto na posição horizontal (litotomia).

Houve correlação entre a posição de parto e episiotomia $(p<0,001)$ sendo essa mais frequente em mulheres que realizaram parto na posição horizontal. Não houve correlação estatisticamente significativa entre posição de parto e uso de ocitocina $(p=0,4)$, ocorrência de laceração perineal $(p=0,3)$ e ocorrência de sutura perineal $(p=0,6)$. (Tabela 1$)$

As características dos neonatos, de acordo com a posição de parto vaginal vertical ou horizontal, estão descritas na Tabela 2. Não houve influência da posição de parto em nenhuma das características dos neonatos: idade gestacional ao nascer $(p=0,74)$, peso ao nascimento $(p=0,85)$, estatura $(p=0,97)$, perímetro cefálico $(p=0,59)$, Apgar no $1^{\circ}(p=0,85)$ e $5^{\circ}$ minuto $(p=0,17)$. (Tabela 2)

Tabela 2

Características neonatais de 176 recém-nascidos de mulheres que realizaram partos vaginais nas posições horizontal e vertical.

\begin{tabular}{|c|c|c|c|c|c|c|c|}
\hline \multirow[t]{2}{*}{ Variáveis neonatais } & \multicolumn{3}{|c|}{ Parto horizontal } & \multicolumn{3}{|c|}{ Parto vertical } & \multirow[t]{2}{*}{$p$} \\
\hline & Intervalo & Média & $\mathrm{DP}$ & Intervalo & Média & DP & \\
\hline Idade gestacional (semanas) & $37-40$ & 38,8 & 0,9 & $37-40$ & 38,9 & 0,9 & 0,74 \\
\hline Peso ao nascimento (g) & $2.130-4.175$ & 3.134 & 409 & $2.330-4.020$ & 3.143 & 349 & 0,85 \\
\hline Estatura $(\mathrm{cm})$ & $42-56$ & 49,6 & 2,4 & $44-56$ & 49,4 & 2,6 & 0,97 \\
\hline Perímetro cefálico $(\mathrm{cm})$ & $30-36$ & 33,5 & 1,4 & $30-37$ & 33,3 & 1,5 & 0,59 \\
\hline Apgar $1^{\circ}$ minuto & $6-10$ & 9 & 1 * & $4-10$ & 9 & 0 * & 0,85 \\
\hline Apgar $5^{\circ}$ minuto & $8-10$ & 10 & 1 * & $7-10$ & 10 & 0 * & 0,17 \\
\hline
\end{tabular}

* Mediana e intervalo interquartil. 


\section{Discussão}

Houve associação entre a posição horizontal de parto vaginal e a ocorrência de episiotomia, mas não entre essa posição e a ocorrência de laceração perineal espontânea. Resultados semelhantes foram encontrados em um ensaio clínico randomizado que investigou a influência da posição materna durante o parto vaginal sobre variáveis obstétricas e neonatais. ${ }^{16}$ Por outro lado, uma metanálise investigando os riscos e benefícios de diversas posições adotadas durante o segundo estágio do trabalho de parto encontrou maior ocorrência de laceração perineal de $2^{\circ}$ grau nos partos verticais ou em decúbito lateral, comparados aos partos horizontais. No entanto, os autores sugerem cautela na interpretação desses resultados devido à qualidade variável dos estudos, inconsistência entre eles e heterogeneidade dos participantes. 11 A associação entre episiotomia e posição horizontal de parto sugere que esse procedimento tenha sido menos necessário durante a realização de partos na posição vertical, em decorrência do efeito facilitador da força da gravidade. Por outro lado, tal resultado pode ser decorrente da dificuldade técnica de se realizar episiotomia em posições verticais de parto vaginal. Independente disso, esses resultados sugerem que a posição vertical de parto vaginal seja um fator protetor para a ocorrência de episiotomia.

A prevalência de lacerações perineais de $3^{\circ}$ grau encontrada na amostra, embora similar aos estudos de Nakai et al.7 e Poen et al., 17 foram inferiores a de outros estudos, que apontam prevalências de até 13\%.18,19 Tal resultado pode ser decorrente da política institucional do hospital onde foi realizado o estudo, que faz uso seletivo da episiotomia, já que o uso rotineiro da episiotomia favorece o surgimento de traumas perineais mais severos. 7,20 O Ministério da Saúde do Brasil21 recomendou, em 2001, uma prevalência de episiotomia entre 10 a $30 \%$ do total dos partos vaginais, valores esses recomendados também por Carroli e Belizan, ${ }^{22}$ após uma revisão sistemática sobre episiotomia. A prevalência de episiotomia na amostra investigada esteve próxima ao limite inferior recomendado. Tais resultados indicam qualidade na assistência obstétrica prestada às mulheres que compuseram a amostra.

A prevalência do uso da ocitocina, documentada no presente estudo, foi inferior à relatada em outros estudos, onde ocitocina foi empregada em até $70 \%$ das vezes. ${ }^{23,24}$ Como a infusão intravenosa de ocitocina é indicada na presença de distócia ou para acelerar o parto, pode trazer como consequência, o desprendimento cefálico abrupto, com maior risco de laceração perineal. ${ }^{2} \mathrm{O}$ uso de ocitocina tem sido também associado à maior prevalência de partos operatórios, sofrimento fetal agudo, além de restringir a movimentação materna durante o primeiro estágio do trabalho de parto. ${ }^{23-26}$ A relativa baixa prevalência do uso de ocitocina na amostra investigada indica que as mulheres atendidas estiveram expostas a menores riscos para tais ocorrências.

Uma das preocupações das mulheres em relação à escolha da posição e tipo de parto refere-se à segurança oferecida para a saúde dos neonatos. Apesar das posições verticais apresentarem o potencial de diminuir a compressão aorto-cava e aumentar a condição metabólica fetal,12 a maioria das mulheres nas sociedades ocidentais realiza parto em decúbito dorsal ou litotomia, alegando-se que tais posições permitem o melhor monitoramento da frequência cardíaca fetal e, consequentemente, asseguram um parto mais seguro do que em posições verticais. 11 Os resultados do presente estudo indicam que as diferentes posições maternas adotadas no segundo estágio de trabalho de parto não interferiram no bemestar fetal. No entanto, futuros estudos, usando métodos mais fidedignos para avaliação da condição metabólica fetal no momento do parto, como a gasometria do cordão umbilical ao nascimento, são necessários para uma posição definitiva sobre o assunto. 27

Em conjunto, os resultados do presente estudo refletem a política institucional do Hospital Sofia Feldman de uso seletivo da episiotomia; sugerem que a posição de parto vaginal vertical minimiza a ocorrência de episiotomia, e indicam que a posição de parto, quando adequadamente indicada, não influencia o estado geral do neonato.

\section{Agradecimentos}

Os autores agradecem ao pessoal do SAME e do CNP/Hospital Sofia Feldman e ao estudante de graduação em Fisioterapia Leandro Magalhães, pelo apoio no levantamento dos dados. 


\section{Referências}

1. Phillips C, Monga A. Childbirth and the pelvic floor: the gynaecological consequences. Rev Gynaecol Pract. 2005; 5: $15-22$.

2. Scarabotto LB, Riesco MLG. Fatores relacionados ao trauma perineal no parto normal em nulíparas. Rev Esc Enferm USP. 2006; 40: 389-95.

3. Casey BM, Schffer JI, Bloom SL, Heartwell SF, McIntire DD, Leveno KJ. Obstetric antecedents for postpartum pelvic floor dysfunction. Am J Obstet Gynecol. 2005; 192: 1655-62.

4. Rortveit G, Dalrveit AK, Hannestad HS. Urinary incontinence after vaginal delivery or cesarean section. $\mathrm{N}$ Engl J Med. 2003; 348: 900-7.

5. Dietz HP, Schierlitz L. Pelvic floor trauma and childbirthmyth or reality? Aust N Z J Obstet Gynaecol. 2005; 45: 311.

6. Dmochowski RR. Urinary incontinence: proper assessment and available treatment options. J Womens Health. 2005; 14: 906-16.

7. Nakai A, Yoshida A, Yamaguchi S, Kawabata I, Hayashi M, Yokota A, Isozaki T, Takeshita T. Incidence and risk factors for severe perineal laceration after vaginal delivery in Japanese patients. Arch Gynecol Obstet. 2006; 274: 222-6.

8. Soong B, Barnes M. Maternal position at midwife-attended birth and perineal trauma: is there an association? Birth. 2005; 32: 164-9.

9. Shorten A, Donsante J, Shorten B. Birth position, accoucheur, and perineal outcomes: informing women about choices for vaginal birth. Birth. 2002; 29: 18-27.

10. Golara M, Plaat F, Shennan AH. Upright versus recumbent position in the second stage of labor in women with combined spinal-epidural analgesia. Int J Obstet Anesth. 2002; 11: 19-22.

11. Gupta JK, Hofmeyr GJ, Smyth R. Position in the second stage of labour for women without epidural anaesthesia. Cochrane Database of Systematic Reviews. 2004; Issue 1. Art. No.: CD002006.

12. Humphrey MD, Chang A, Wood EC, Morgan S, Hounslow D. A decrease in fetal $\mathrm{pH}$ during the second stage of labour when conducted in the dorsal position. J Obstet Gynaecol Br Commow. 1974; 81: 600-2.

13. Mamede FV, Almeida AM, Clapis MJ Movimentação/deambulação no trabalho de parto: uma revisão. Acta Sci. 2004; 26: 295-302.

14. Royal College of Obstetricians and Gynaecologists. Management of third- and fourth-degree perineal tears following vaginal delivery. Guideline $\mathrm{n}^{\mathrm{o}}$. 29. July; 2001.
15. Cohen J. Statistical power analysis for the behavioral sciences. 2 ed. New Jersey: Lawrence Erlbaum; 1988.

16. Bonfim-Hyppólito S. Influence of the position of the mother at delivery over some maternal and neonatal outcomes. Int J Gynecol Obstet. 1998; 63: 67-73.

17. Poen AC, Felt-Bersma RJ, Dekker GA, Devillé W, Cuesta MA, Meuwissen SG. Third degree obstetric perineal tears: risk factors and the preventive role of mediolateral episiotomy. Br J Obstet Gynaecol. 1997; 104: 563-6.

18. Andrews V, Sultan AH, Thakar R, Jones PW. Risk factors for obstetric anal sphincter injury: a prospective study. Birth. 2006; 33: 117-22.

19. Goldberg J, Hyslop T, Tolosa JE, Sultana C. Racial differences in severe perineal lacerations after vaginal deleivery. Am J Obstet Gynecol. 2003; 188: 1063-7.

20. Clemons JL, Towers GD, McClure GB, O'Boyle AL. Decreased anal sphincter lacerations associated with restrictive episiotomy use. Am J Obstet Gynecol. 2005; 192: 1620-5.

21. Brasil. Ministério da Saúde. Secretaria de Políticas de Saúde. Área Técnica de Saúde da Mulher. Parto, aborto e puerpério: assistências humanizadas à mulher. Brasília, DF; 2001

22. Carroli G, Belizan J. Episiotomy for vaginal birth. Cochrane Database of Systematic Reviews. 1999; Issue 3. Art. N.: CD000081.

23. Svärdby K, Nordström L, Sellström E. Primiparas with or without oxytocin augmentation: a prospective descriptive study. J Clin Nurs. 2007; 16: 179-84

24. Oscarsson Me, Amer-Wahlin I, Rydhstroem H, Källén K Outcome in obstetric care related to oxytocin use. A population-based study. Acta Obstet Gynecol Scand. 2006; 85: 1094-8.

25. Thornton JG, Lilford RJ. Active management of labour current knowledge and research issues. BMJ. 1994; 339: 1336-8.

26. Bloom SL, McIntire DD, Kelly MA, Beimer HL, Burpo RH, Garcia MA, Leveno KJ. Lack of effect of walking in labour and delivery. N Engl J Med. 1994; 339: 76-9.

27. ACOG. (American College of Obstetricians and Gynecologists). Committee Opinion Number 348. Umbilical cord blood gas and acid-base analysis. Obstet Gynecol. 2006; 108: 1319-22.

Recebido em 8 de outubro de 2008

Versão final apresentada em 3 de agosto de 2009

Aprovado em 28 de setembro de 2009 